Authors' financial interests should be made known to manuscript reviewers

SIR - Much emphasis has been put on the importance of policies that require authors to be transparent about financial conflicts of interest. Nature, for example, requires most authors to submit a declaration of any competing financial interests in relation to the work described in a submitted article. The reason why journals have these policies is, presumably, to safeguard the objectivity of the research. Transparency is thought to promote objectivity because if readers are aware of potential financial conflicts, they can critically evaluate the ways in which such interests may have affected the research - for example, in the selection of evidence, interpretation of results, or research methodology.

Yet transparency is insufficient as a safeguard of objectivity. Scientific expertise is necessary to correctly evaluate whether conflicts have biased the research, yet financial conflicts are revealed only when an article is published. This prevents peerreviewers - who are in the best position to evaluate the possible influence of the conflicts of interest - from having access to the information. Thus, it is not clear to us how revealing financial interests in a statement accompanying publicatio of an article can allow readers to make accurate assessments of bias.

In addition, these policies foster an abrogation of scientific responsibility by the research community, because they put the burden of critical evaluation on the public, who in the main are not scientifically knowledgeable at a detailed level. This aspect is of particular concern for papers in journals such as Nature, which are likely to be widely disseminated to the public by the media. Even if biases are identified after publication and a correction is made, such criticisms tend not to be publicized to the same extent as the original article.

If the aim of conflict-of-interest policies is to promote objectivity and inform readers and the public, we believe a more effective approach would be for authors to be required to reveal possible financial competing interests, not only to the public after publication, but also to reviewers during the peer-review process.

Inmaculada de Melo-Martín ${ }^{\star}$, Kristen Intemann'† ${ }^{\star}$ Division of Medical Ethics, Department of Public Health, Weill Cornell Medical College, 411 East 69th Street, New York, New York 10021, USA $\uparrow$ Department of History and Philosophy, Montana State University, 2-155 Wilson Hall, Bozeman, Montana 59717, USA

Comments on the suggestion made in this Correspondence, and on Nature's policy, are welcome at Peer to Peer, the blog for and about peer review, at http://blogs.nature. com/peer-to-peer/2007/07/should_ authors_financial_inter.html

\section{Space-time safe - at least until the LHC switches on}

SIR - You are either wildly optimistic, or confused, to suggest in your News Feature 'Extreme light' (Nature 446, $16-18 ; 2007)$ that extreme laser experiments could "rip apart the fabric of space and time".

The phenomena of Unruh radiation and Schwinger pair production, which are the motivation for extreme laser experiments, simply probe the behaviour of quantum fields on a fixed, smooth spacetime. Even if the experiments are totally successful, the only thing that could be 'ripped apart' is the vacuum state of some quantum fields.

If one considers quantum theories of gravity, there is no formal way to distinguish the space-time manifold from the quantum fields, because space-time itself should be quantized. (Don't ask me how.) But at low momenta and energy densities, relative to the fundamental scale of quantum gravity, usually around the Planck scale of $10^{19} \mathrm{GeV}$, such theories should reduce to something like the well-known picture of quantum field theory on a classical, weakly curved manifold described by general relativity, which has been successful in describing particle physics and optics.

Hence it is very difficult to probe quantum gravity experimentally, unless the low-energy limit of the theory gives some (necessarily small) deviation from this established picture. For example, varying 'constants', equivalence principle violations or Lorentz violation could amount to an indirect probe.

There are also special classes of theories in which the fundamental scale of quantum gravity may be much lower, even a few thousand GeV. However, these extra dimensions would not be probed by laser experiments, since photons do not propagate along them, unless the energy density approached the fundamental $\left(\mathrm{TeV}^{4}\right)$ scale. Whether an exawatt beam (rated by power, rather than energy density) could do so depends on its focusing and pulse length: I highly doubt it.

The main hope at present to probe theories with $\mathrm{TeV}$ fundamental scale comes from the Large Hadron Collider (LHC), which is designed to produce such large energy densities in some of its collisions.

It is conceivable that black holes might result from gravitational collapse of dense regions - which would, finally, be a tale of ripping the fabric of space-time. Before the LHC switches on, space-time should be quite safe. Depending on your point of view, this may be a source either of relief or of disappointment.

Thomas Dent

Theoretical Physics, University of Heidelberg, Philosophenweg 16, 69120 Heidelberg, Germany

\section{Mentors could support a student reviewer database}

SIR - Your Editorial 'Mentors of the future' (Nature 447, 754; 2007) highlights a need to encourage ethical, honest and fair peer review by young scientists. Although I applaud the ethos of the argument presented, graduate students such as myself often suffer from anonymity in their field of research, even though our work is often at the cutting edge. A lack of publications can render a student invisible to editors and may result in missed opportunities to offer their services as referees.

I suggest that journals consider introducing a 'PhD student peer-review pool' to which students and their supervisors can sign up. Such a database, including a student's name, area of research expertise and current supervisors, would provide editors with a ready supply of willing referees. Editors could try new referees in the knowledge that they will be supported during the review process by their supervisor, and could provide feedback to the student about the quality of the report.

Refereeing has often been described as a thankless task, but although it does require considerable effort, it also provides invaluable experience in critical interpretation of science. Having recently completed my first review, I believe that it has made me far more objective about my own writing and can only benefit the production of my thesis. I therefore look forward to receiving my next invitation to review.

Angelo P. Pernetta

Centre for Ecology and Hydrology, FBA East Stoke, Wareham, Dorset BH20 6BB, UK

\section{Mentors: public lists would help students choose}

SIR - Thank you for your excellent Feature, 'Nature's guide for mentors' (Nature 447, 791-797; 2007). It's definitely one of the best things I've read in Nature in the 25 years I've been reading the journal. To better help students make informed choices about choosing a mentor, it would be enormously useful if public granting agencies such as the US National Institutes of Health would 
publicly post the 'trainees' lists that are included in training grants for every faculty member in a given $\mathrm{PhD}$ training programme. If this were done, students considering applying to those labs would know their actual chance of being mentored successfully. Ben Barres

Department of Neurobiology, Stanford University School of Medicine, 299 Campus Drive, Stanford, California 94305-5125, USA

\section{How incompatibilities may have led to eukaryotic cell}

SIR - In the Connections Essay 'Disappearing act' (Nature 446, 983; 2007), James A. Lake discusses the evolution of the eukaryotic cell from the perspective of the origin of the eukaryotic gene content. As he points out, there are two main gene classes in prokaryotes (archaea and bacteria): operational genes, for "day-to-day processes of cell maintenance"; and informational genes, that "convert information from DNA into proteins". Eukaryotes are derived from archaea and bacteria, but curiously, archaeal operational genes and bacterial informational genes are almost completely absent from the eukaryotic genome. Lake suggests that "because two types of ribosomal genes cannot exist in the same nucleus, the archaebacterial ribosome may simply have been the lucky survivor when one of the components in the eubacterial ribosome was inactivated." But, Lake asks his readers, why were the archaebacterial operational genes eliminated?

One hypothesis is that the archaeal and bacterial cells that merged to form the eukaryotic cell were 'metabolically incompatible', and consequently their merger resulted in the elimination of the archaeal operational genes. But what, then, was the basis for this 'metabolic incompatibility' and for the bacterial genes to finally get the upper hand? David Valentine has suggested that adaptations to energy stress dictate the ecology and evolution of archaea (Nature Rev. Microbiol. 5, 316-323; 2007). The biochemical mechanisms enabling archaea to cope with chronic energy stress include structural (a less ion-permeable membrane) and metabolic (pathways highly adapted to niches with low energy availability) components.

We suggest that during eukaryogenesis, the bacterial endosymbiont, which gradually became the mitochondrion, ended the chronic energy stress in the proto-eukaryote. In contrast to the anaerobic archaeal host, the aerobic bacterial endosymbiont was able to maximize the availability of energy and gradually become an efficient energyconverting organelle, the 'powerhouse' of the eukaryotic cell. The proto-eukaryotic metabolism had to be reorganized accordingly. The unique archaeal adaptations to chronic energy stress were no longer advantageous, and were out-competed by the 'higher energy'-adapted bacterial metabolism. Lateral gene transfer from organisms other than the two founders certainly contributed to the eukaryotic gene repertoire, but we think that operational genes mostly originated from bacterial donors because the archaeal genes were incompatible with the energy-rich environment of the eukaryotic cell.

To return to Lake's Essay, the two faces of the eukaryotic gene content (the Janus paradox) might reflect two types of incompatibilities between its two prokaryotic founders: structural incompatibility between the informational systems; and environmental (or ecological) incompatibility between the metabolic systems. These eventually led to the synergetic, two-faced, chimaeric result the eukaryotic cell.

Yaacov Davidov^, Edouard Jurkevitch $†$

*Department of Biological Chemistry,

The Weizmann Institute of Science,

76100 Rehovot, Israel

†Department of Plant Pathology and

Microbiology, The Hebrew University of

Jerusalem, 76100 Rehovot, Israel

\section{Text mining: powering the database revolution}

SIR - Mark Gerstein and colleagues in Correspondence (Nature 447, 142; 2007) propose that journals should require authors to manually provide structured abstracts to facilitate text mining of biological information. There are three main difficulties in implementing such a proposal.

First, life-science terminologies are huge, diversified and complex. This means that identifying the correct content descriptors is almost impossible for inexperienced users of online term repositories. For example, Medical Subject Headings (www.nlm.nih. gov/mesh), the International Classification of Diseases (www.who.int/classifications/icd/ en) and Gene Ontology (www.geneontology. org) are high-volume - tens of thousands of terms - and structurally complicated terminological systems, each with different design rationales, naming conventions and principles of structural organization. Even human indexers, search specialists and database curators with routine exposure to these resources have to invest much effort in understanding and keeping track of their content as well as terminological updates and revisions. Will scientists find the time to dive so deeply into this alien terminological territory, and be capable of finding exactly what they are looking for?

Second, the coverage of existing terminologies for the many subdomains in the life sciences is incomplete. The two main terminological umbrella systems for the life sciences, the Unified Medical Language System (http://umlsinfo.nlm.nih.gov) and the Open Biomedical Ontologies (http://obo.sourceforge.net/main.html), contain impressive numbers of individual terminologies, but their coverage of the life sciences is still fragmentary and suffers from varying depths of description. The size of the terminology gap is likely to be even more pronounced if authors were required to encode relational descriptions, for example indicating a binding relation between two specific proteins, $\mathrm{P} 1$ and $\mathrm{P} 2$, by $\operatorname{Bind}(\mathrm{P} 1, \mathrm{P} 2)$, because such a vocabulary has not yet been determined.

Third, the quality and reliability of authorsupplied content descriptions is quite a hurdle. Even if the first and second problems were to be solved, human indexers, even professional ones, are liable to error as well as to the possibility of intrinsic subjective bias (M. E. Funk and C. A. Reid Bull. Med. Libr. Assoc. 71, 176-183; 1983). This is not to say that authors of a structured abstract would consciously cheat, but rather there is a grey area of overstatement and overestimation of one's own results in a highly competitive scientific environment. If authors' structured entries were subject to peer review together with the submitted article, this would be more work for the reviewers as well as the authors - neither of them likely to have been trained as terminologists.

As an alternative, we suggest automated procedures for knowledge capture in which neither the authors nor the reviewers are in the loop. There has been significant progress in automatic text mining and information extraction as well as in the methodological foundations of life-science terminologies in terms of ontologies, knowledge representation languages and semantic encoding standards. These efforts in automating the generation of content descriptions and linking them directly to biological databases are strongly experimentally founded and would help to avoid additional workload and subjectivity see, for example, the BioCreAtIvE competition results (http://biocreative. sourceforge.net). Once automated mechanisms for content analysis are applied, this also increases the coverage and the recency of the literature entered into biological databases, as human input is complemented by computationally generated content.

Udo Hahn`, Joachim Wermter *,

Rainer Blasczyk $\dagger$, Peter A. Horn'†

*Friedrich Schiller University Jena,

Computational Linguistics Group - JULIE Lab,

07743 Jena, Germany

† Institute for Transfusion Medicine,

Hannover Medical School,

30625 Hannover, Germany 